\title{
Toeplitz and Translation Operators on the q-Fock Spaces*
}

\author{
Fethi Soltani \\ Higher College of Technology and Informatics, Tunis, Tunisia \\ E-mail: fethisoltani10@yahoo.com
}

Received June 25, 2011; revised July 12, 2011; accepted July 28, 2011

\section{Abstract}

In this work, we introduce a class of Hilbert spaces $\mathcal{F}_{q}$ of entire functions on the disk $D\left(o, \frac{1}{\sqrt{1-q}}\right)$, $0<q<1$, with reproducing kernel given by the $q$-exponential function $e_{q}(z)$; and we prove some properties concerning Toeplitz operators on this space. The definition and properties of the space $\mathcal{F}_{q}$ extend naturally those of the well-known classical Fock space. Next, we study the multiplication operator $Q$ by $Z$ and the $q$-Derivative operator $D_{q}$ on the Fock space $\mathcal{F}_{q}$; and we prove that these operators are adjoint-operators and continuous from this space into itself. Lastly, we study a generalized translation operators and a Weyl commutation relations on $\mathcal{F}_{q}$.

Keywords: $q$-Fock Spaces, $q$-Exponential Function, $q$-Derivative Operator, $q$-Translation Operators, $q$-Toeplitz Operators, $q$-Weyl Commutation Relations

\section{Introduction}

In 1961, Bargmann [1] introduced a Hilbert space $\mathcal{F}$ of entire functions $f(z)=\sum_{n=0}^{\infty} a_{n} z^{n}$ on $\mathbb{C}$ such that

$$
\|f\|_{\mathcal{F}}^{2}:=\sum_{n=0}^{\infty}\left|a_{n}\right|^{2} n !<\infty \text {. }
$$

On this space the author study the differential operator $D=\mathrm{d} / \mathrm{d} z$ and the multiplication operator by $z$, and proves that these operators are densely defined, closed and adjoint-operators on $\mathcal{F}$ (see [1]). Next, the Hilbert space $\mathcal{F}$ is called Segal-Bargmann space or Fock space and it was the aim of many works $[2,3]$.

In this paper, we consider the $q$-exponential function:

$$
e_{q}(z):=\sum_{n=0}^{\infty} \frac{(1-q)^{n}}{(q ; q)_{n}} z^{n}
$$

where

$$
(q ; q)_{n}:=\prod_{i=0}^{n-1}\left(1-q^{i+1}\right), \quad n=1,2, \cdots, \infty .
$$

We discuss some properties of a class of Fock spaces

"Author partially supported by DGRST project 04/UR/15-02 and CMCU program 10G 1503. associated to the $q$-exponential function and we give some applications.

In the first part of this work, building on the ideas of Bargmann [1], we define the $q$-Fock space $\mathcal{F}_{q}$ as the space of entire functions $f(z)=\sum_{n=0}^{\infty} a_{n} z^{n}$ on the disk $D\left(o, \frac{1}{\sqrt{1-q}}\right)$ of center $o$ and radius $\frac{1}{\sqrt{1-q}}$, and such that

$$
\|f\|_{\mathcal{F}_{q}}^{2}:=\sum_{n=0}^{\infty}\left|a_{n}\right|^{2} \frac{(q ; q)_{n}}{(1-q)^{n}}<\infty .
$$

Let $f$ and $g$ be in $\mathcal{F}_{q}$, such that $f(z)=\sum_{n=0}^{\infty} a_{n} z^{n}$ and $g(z)=\sum_{n=0}^{\infty} b_{n} z^{n}$, the inner product is given by

$$
\langle f, g\rangle_{\mathcal{F}_{q}}=\sum_{n=0}^{\infty} a_{n} \overline{b_{n}} \frac{(q ; q)_{n}}{(1-q)^{n}} .
$$

The $q$-Fock space $\mathcal{F}_{q}$ has also a reproducing kernel $\mathcal{K}_{q}$ given by

$$
\mathcal{K}_{q}(w, z)=e_{q}(\bar{w} z) ; \quad w, z \in D\left(o, \frac{1}{\sqrt{1-q}}\right) .
$$


Then if $f \in \mathcal{F}_{q}$, we have

$$
\left\langle f, \mathcal{K}_{q}(w, .)\right\rangle_{\mathcal{F}_{q}}=f(w), \quad w \in D\left(o, \frac{1}{\sqrt{1-q}}\right) .
$$

Using this property, we prove that the space $\mathcal{F}_{q}$ is a Hilbert space and we give an Hilbert basis.

Next, we define and study the Toeplitz operators of the $q$-Fock space $\mathcal{F}_{q}$.

In the second part of this work, we consider the multiplication operator $Q$ by $z$ and the $q$-Derivative operator $D_{q}$ on the Fock space $\mathcal{F}_{q}$, and we prove that these operators are continuous from $\mathcal{F}_{q}$ into itself, and satisfy:

$$
\left\|D_{q} f\right\|_{\mathcal{F}_{q}} \leq \frac{1}{\sqrt{1-q}}\|f\|_{\mathcal{F}_{q}}, \quad\|Q f\|_{\mathcal{F}_{q}} \leq \frac{1}{\sqrt{1-q}}\|f\|_{\mathcal{F}_{q}} .
$$

Then, we prove that these operators are adjoint-operators on $\mathcal{F}_{q}$ :

$$
\langle Q f, g\rangle_{\mathcal{F}_{q}}=\left\langle f, D_{q} g\right\rangle_{\mathcal{F}_{q}} ; \quad f, g \in \mathcal{F}_{q} .
$$

Next, we define and study on the Fock space $\mathcal{F}_{q}$, the $q$-translation operators:

$$
\tau_{z} f(w):=e_{q}\left(z D_{q}\right) f(w) ; \quad w, z \in D\left(o, \frac{1}{\sqrt{1-q}}\right),
$$

and the generalized multiplication operators:

$$
M_{z} f(w):=e_{q}(z Q) f(w) ; \quad w, z \in D\left(o, \frac{1}{\sqrt{1-q}}\right) .
$$

Using the previous results, we deduce that the operators $\tau_{z}$ and $M_{z}$, for $z \in D\left(o, \frac{1}{\sqrt{1-q}}\right)$, are continuous from $\mathcal{F}_{q}$ into itself, and satisfy:

$$
\begin{aligned}
& \left\|\tau_{z} f\right\|_{\mathcal{F}_{q}} \leq e_{q}\left(\frac{|z|}{\sqrt{1-q}}\right)\|f\|_{\mathcal{F}_{q}}, \\
& \left\|M_{z} f\right\|_{\mathcal{F}_{q}} \leq e_{q}\left(\frac{|z|}{\sqrt{1-q}}\right)\|f\|_{\mathcal{F}} .
\end{aligned}
$$

Lastly, we establish Weyl commutation relations between the translation operators $\tau_{a}$ and the multiplication operators $M_{b}$, where $a, b \in D\left(o, \frac{1}{\sqrt{1-q}}\right)$. These relations are realized on the Fock space $\mathcal{F}_{q}$.

\section{The $q$-Fock Spaces $\mathcal{F}_{q}$ and the Toeplitz Operators}

\subsection{Preliminaries}

Let $a$ and $q$ be real numbers such that $0<q<1$; the $q$-shifted factorial are defined by

$$
(a ; q)_{0}:=1,(a ; q)_{n}:=\prod_{i=0}^{n-1}\left(1-a q^{i}\right), n=1,2, \cdots, \infty .
$$

Jackson [4] defined the $q$-analogue of the Gamma function as

$$
\Gamma_{q}(x):=\frac{(q ; q)_{\infty}}{\left(q^{x} ; q\right)_{\infty}}(1-q)^{1-x}, \quad x \neq 0,-1,-2, \cdots
$$

It satisfies the functional equation

$$
\Gamma_{q}(x+1)=[x]_{q} \Gamma_{q}(x), \quad \Gamma_{q}(1)=1,
$$

where

$$
[x]_{q}:=\frac{1-q^{x}}{1-q}
$$

and tends to $\Gamma(x)$ when $q$ tends to $1^{-}$. In particular, for $n=1,2, \cdots$, we have

$$
\Gamma_{q}(n+1)=\frac{(q ; q)_{n}}{(1-q)^{n}}=[n]_{q} ! .
$$

The $q$-combinatorial coefficients are defined for $n \in \mathbb{N}$ and $k=0, \cdots, n$, by

$$
\left(\begin{array}{l}
n \\
k
\end{array}\right)_{q}:=\frac{[n]_{q} !}{[k]_{q} ![n-k]_{q} !} .
$$

The $q$-derivative $D_{q} f$ of a suitable function $f$ (see [5]) is given by

$$
D_{q} f(x):=\frac{f(x)-f(q x)}{(1-q) x}, \quad x \neq 0,
$$

and $D_{q} f(0)=f^{\prime}(0)$ provided $f^{\prime}(0)$ exists.

If $f$ is differentiable then $D_{q} f(x)$ tends to $f^{\prime}(x)$ as $q \rightarrow 1^{-}$.

There are two important $q$-analogues of the exponential function [5]:

$$
\begin{gathered}
E_{q}(z):=\sum_{n=0}^{\infty} q^{n(n-1) / 2} \frac{z^{n}}{[n]_{q} !}, \\
e_{q}(z):=\sum_{n=0}^{\infty} \frac{z^{n}}{[n]_{q} !}
\end{gathered}
$$

Note that the first series converges for $|z|<\infty$ and the second series converges for $|z|<\frac{1}{1-q}$. 
Therefore the function $\Gamma_{q}$ has the $q$-integral representation [6]:

$$
\Gamma_{q}(x)=\int_{0}^{\frac{1}{1-q}} r^{x-1} E_{q}(-q r) \mathrm{d}_{q} r, \quad x>0,
$$

where the $q$-integral (introduced by Jackson [4]) is defined by

$$
\int_{0}^{a} f(x) \mathrm{d}_{q} x=(1-q) \sum_{n=0}^{\infty} a q^{n} f\left(a q^{n}\right) .
$$

Lemma 1. The function $e_{q}(\lambda),. \lambda \in D\left(o, \frac{1}{\sqrt{1-q}}\right)$, is the unique analytic solution of the q-problem:

$$
D_{q} y(z)=\lambda y(z), \quad y(0)=1 .
$$

Proof. Searching a solution of (2) in the form $y(z)=\sum_{n=0}^{\infty} a_{n} z^{n}$. Then

$$
D_{q} y(z)=\sum_{n=1}^{\infty} a_{n}[n]_{q} z^{n-1} .
$$

Replacing in (2), we obtain

$$
\sum_{n=1}^{\infty} a_{n}[n]_{q} z^{n}=\lambda \sum_{n=1}^{\infty} a_{n-1} x^{n}
$$

Thus,

$$
a_{n}[n]_{q}=\lambda a_{n-1}, \quad n=1,2, \cdots
$$

We deduce that

$$
a_{n}=\frac{\lambda}{[n]_{q}} a_{n-1}
$$

We get

$$
a_{n}=\frac{\lambda^{n}}{[n]_{q} !}
$$

Therefore,

$$
y(z)=\sum_{n=0}^{\infty} \frac{(\lambda z)^{n}}{[n]_{q} !}=e_{q}(\lambda z),
$$

which completes the proof of the lemma.

\subsection{The $q$-Fock Spaces $\mathcal{F}_{q}$}

We denote by

- $H\left(D\left(o, \frac{1}{\sqrt{1-q}}\right)\right)$ the space of entire functions on $D\left(o, \frac{1}{\sqrt{1-q}}\right)$.
- $m_{q}$ the measure defined on $D\left(o, \frac{1}{\sqrt{1-q}}\right)$ by

$$
d m_{q}(z):=\frac{1}{2 \pi} E_{q}(-q r) d_{q} r d \theta, \quad z=\sqrt{r} \mathrm{e}^{i \theta} .
$$

- $L^{2}\left(D\left(o, \frac{1}{\sqrt{1-q}}\right), m_{q}\right)$ the space of measurable functions $f$ on $D\left(o, \frac{1}{\sqrt{1-q}}\right)$ satisfying

$$
\|f\|_{L^{2}\left(D\left(o, \frac{1}{\sqrt{1-q}}\right), m_{q}\right)}^{2}:=\int_{D\left(o, \frac{1}{\sqrt{1-q}}\right)}|f(z)|^{2} d m_{q}(z)<\infty .
$$

Definition 1. We define the prehilbertian space $\mathcal{F}_{q}$, to be the space of functions in $H\left(D\left(o, \frac{1}{\sqrt{1-q}}\right)\right) \cap L^{2}\left(D\left(o, \frac{1}{\sqrt{1-q}}\right), m_{q}\right)$, equipped with the inner product

$$
\langle f, g\rangle_{\mathcal{F}_{q}}=\int_{D\left(o, \frac{1}{\sqrt{1-q}}\right)} f(z) \overline{g(z)} d m_{q}(z),
$$

and the norm

$$
\|f\|_{\mathcal{F}_{q}}=\left[\int_{D\left(o, \frac{1}{\sqrt{1-q}}\right)}|f(z)|^{2} d m_{q}(z)\right]^{1 / 2} .
$$

Remark 1. If $q \rightarrow 1^{-}$, the space $\mathcal{F}_{q}$ agrees with the Segal-Bargmann's space (see [1]).

Proposition 1. 1) For all $f \in \mathcal{F}_{q}$ such that $f(z)=\sum_{n=0}^{\infty} a_{n} z^{n}$, we have

$$
\|f\|_{\mathcal{F}_{q}}^{2}=\sum_{n=0}^{\infty}\left|a_{n}\right|^{2}[n]_{q} !
$$

2) For all $f, g \in \mathcal{F}_{q}$ such that $f(z)=\sum_{n=0}^{\infty} a_{n} z^{n}$ and $g(z)=\sum_{n=0}^{\infty} b_{n} z^{n}$, we have

$$
\langle f, g\rangle_{\mathcal{F}_{q}}=\sum_{n=0}^{\infty} a_{n} \overline{b_{n}}[n]_{q} !
$$

3) For $f, g \in \mathcal{F}_{q}$, we have

$$
\langle f, g\rangle_{\mathcal{F}_{q}}=f\left(D_{q}\right) \tilde{g}(0), \quad \tilde{g}(z)=\overline{g(\bar{z})} .
$$

Proof. Given $f(z)=\sum_{n=0}^{\infty} a_{n} z^{n} \in \mathcal{F}_{q}$ and $g(z)=\sum_{n=0}^{\infty} b_{n} z^{n} \in \mathcal{F}_{q}$.

1) By dominated convergence theorem's, we have

$$
\|f\|_{\mathcal{F}_{q}}^{2}=\sum_{m, n=0}^{\infty} a_{m} \overline{a_{n}} \int_{D\left(o, \frac{1}{\sqrt{1-q}}\right)} z^{m} \bar{Z}^{-n} d m_{q}(z) .
$$


We put $z=\sqrt{r} \mathrm{e}^{i \theta}$, then we deduce

$$
\|f\|_{\mathcal{F}_{q}}^{2}=\sum_{n=0}^{\infty}\left|a_{n}\right|^{2} \int_{0}^{\frac{1}{1-q}} r^{n} E_{q}(-q r) \mathrm{d}_{q} r .
$$

But from (1), we have

$$
\int_{0}^{\frac{1}{1-q}} r^{n} E_{q}(-q r) \mathrm{d}_{q} r=\Gamma_{q}(n+1)=[n]_{q} ! .
$$

Thus,

$$
\|f\|_{\mathcal{F}_{q}}^{2}=\sum_{n=0}^{\infty}\left|a_{n}\right|^{2}[n]_{q} ! .
$$

2) We obtain the result from (1) by polarization.

3) Since

$$
D_{q} z^{k}=[k]_{q} z^{k-1}, \quad k \geq 1,
$$

then

$$
D_{q}^{n} z^{k}=\frac{[k]_{q} !}{[k-n]_{q} !} z^{k-n}, \quad k \geq n,
$$

and

$$
D_{q}^{n} g(z)=\sum_{k=n}^{\infty} \frac{[k]_{q} !}{[k-n]_{q} !} b_{k} z^{k-n}
$$

Thus,

$$
b_{n}=\frac{D_{q}^{n} g(0)}{[n]_{q} !}
$$

and

$$
g(z)=\sum_{n=0}^{\infty} \frac{D_{q}^{n} g(0)}{[n]_{q} !} z^{n} .
$$

Using (4) and (6), we get

$$
\langle f, g\rangle_{\mathcal{F}_{q}}=\sum_{n=0}^{\infty} a_{n} \overline{D_{q}^{n} g(0)}=\sum_{n=0}^{\infty} a_{n} D_{q}^{n} \tilde{g}(0) .
$$

Thus

$$
\langle f, g\rangle_{\mathcal{F}_{q}}=f\left(D_{q}\right) \tilde{g}(0)
$$

which gives the desired result.

The following theorem prove that $\mathcal{F}_{q}$ is a reproducing kernel space.

Theorem 1. The function $\mathcal{K}_{q}$ given for $w, z \in D\left(o, \frac{1}{\sqrt{1-q}}\right)$, by

$$
\mathcal{K}_{q}(w, z)=e_{q}(\bar{w} z)
$$

is a reproducing kernel for the $q$-Fock space $\mathcal{F}_{q}$, that is:
1) for all $w \in D\left(o, \frac{1}{\sqrt{1-q}}\right)$, the function $z \rightarrow \mathcal{K}_{q}(w, z)$ belongs to $\mathcal{F}_{q}$.

2) For all $w \in D\left(o, \frac{1}{\sqrt{1-q}}\right)$ and $f \in \mathcal{F}_{q}$, we have

$$
\left\langle f, \mathcal{K}_{q}(w, .)\right\rangle_{\mathcal{F}_{q}}=f(w) .
$$

Proof. 1) Since

$$
\mathcal{K}_{q}(w, z)=\sum_{n=0}^{\infty} \frac{\bar{w}^{n} z^{n}}{[n]_{q} !} ; \quad z, w \in D\left(o, \frac{1}{\sqrt{1-q}}\right),
$$

then from (3), we deduce that

$$
\left\|\mathcal{K}_{q}(w, .)\right\|_{\mathcal{F}_{q}}^{2}=\sum_{n=0}^{\infty} \frac{|w|^{2 n}}{[n]_{q} !}=e_{q}\left(|w|^{2}\right)<\infty
$$

which proves 1).

2) If $f(z)=\sum_{n=0}^{\infty} a_{n} z^{n} \in \mathcal{F}_{q}$, from (4) and (7), we deduce

$$
\left\langle f, \mathcal{K}_{q}(w, .)\right\rangle_{\mathcal{F}_{q}}=\sum_{n=0}^{\infty} a_{n} w^{n}=f(w), \quad w \in D\left(o, \frac{1}{\sqrt{1-q}}\right) .
$$

This completes the proof of the theorem.

Remark 2. From Theorem 1 (2), for $f \in \mathcal{F}_{q}$ and $w \in D\left(o, \frac{1}{\sqrt{1-q}}\right)$, we have

$$
|f(w)| \leq\left\|\mathcal{K}_{q}(w, .)\right\|_{\mathcal{F}_{q}}\|f\|_{\mathcal{F}_{q}}=\left[e_{q}\left(|w|^{2}\right)\right]^{1 / 2}\|f\|_{\mathcal{F}_{q}} .
$$

Proposition 2. The space $\mathcal{F}_{q}$ equipped with the inner product $\langle., .\rangle_{\mathcal{F}_{q}}$ is an Hilbert space; and the set $\left\{\xi_{n}\right\}_{n \in \mathbb{N}}$ given by

$$
\xi_{n}(z)=\frac{z^{n}}{\sqrt{[n]_{q} !}}, \quad z \in D\left(o, \frac{1}{\sqrt{1-q}}\right),
$$

forms an Hilbert basis for the space $\mathcal{F}_{q}$.

Proof. Let $\left\{\xi_{n}\right\}_{n \in \mathbb{N}}$ be a Cauchy sequence in $\mathcal{F}_{q}$. We put

$$
f=\lim _{n \rightarrow \infty} f_{n}, \quad \text { in } \mathcal{F}_{q} .
$$

From (8), we have

$$
\left|f_{n+p}(w)-f_{n}(w)\right| \leq\left[e_{q}\left(|w|^{2}\right)\right]^{1 / 2}\left\|f_{n+p}-f_{n}\right\|_{\mathcal{F}_{q}} .
$$

This inequality shows that the sequence $\left\{f_{n}\right\}_{n \in \mathbb{N}}$ is pointwise convergent to $f$. Since the function $w \rightarrow\left[e_{q}\left(|w|^{2}\right)\right]^{1 / 2}$ is continuous on $D\left(o, \frac{1}{\sqrt{1-q}}\right)$, then 
$\left\{f_{n}\right\}_{n \in \mathbb{N}}$ converges to $f$ uniformly on all compact set of $D\left(o, \frac{1}{\sqrt{1-q}}\right)$. Consequently, $f$ is an entire function on $D\left(o, \frac{1}{\sqrt{1-q}}\right)$, then $f$ belongs to the space $\mathcal{F}_{q}$.

On the other hand, from the relation (4), we get

$$
\left\langle\xi_{n}, \xi_{m}\right\rangle_{\mathcal{F}_{q}}=\delta_{n, m}
$$

where $\delta_{n, m}$ is the Kronecker symbol.

This shows that the family $\left\{\xi_{n}\right\}_{n \in \mathbb{N}}$ is an orthonormal set in $\mathcal{F}_{q}$.

Let $f(z)=\sum_{n=0}^{\infty} a_{n} z^{n}$ be an element of $\mathcal{F}_{q}$ such that

$$
\left\langle f, \xi_{n}\right\rangle_{\mathcal{F}_{q}}=0, \quad \forall n \in \mathbb{N} .
$$

From the relation (4), we deduce that

$$
a_{n}=0, \quad \forall n \in \mathbb{N} .
$$

This completes the proof.

\subsection{Toepliz Operators on $\mathcal{F}_{q}$}

In this paragraph we study the Toeplitz operators on $\mathcal{F}_{q}$. These operators generalize the classical Toeplitz operators [2].

First we define the orthogonal projection operator $P$ from $L^{2}\left(D\left(o, \frac{1}{\sqrt{1-q}}\right), m_{q}\right)$ into $\mathcal{F}_{q}$, by

$$
\begin{aligned}
& P f(w):=\left\langle f, K_{q}(w, .)\right\rangle_{L^{2}}\left(D\left(o, \frac{1}{\sqrt{1-q}}\right), m_{q}\right), \\
& w \in D\left(o, \frac{1}{\sqrt{1-q}}\right),
\end{aligned}
$$

where $K_{q}$ is the reproducing kernel given by (7).

Definition 2. Let $\phi$ be a measurable function on $D\left(o, \frac{1}{\sqrt{1-q}}\right)$. The Toeplitz operator $T_{\phi}$ is the operator given by

$$
T_{\phi} f:=P(\phi f),
$$

for every

$$
f \in D\left(T_{\phi}\right):=\left\{f \in \mathcal{F}_{q}: \varphi f \in L^{2}\left(D\left(o, \frac{1}{\sqrt{1-q}}\right), m_{q}\right)\right\} .
$$

Remark 3. Let $\phi \in L^{\infty}\left(D\left(o, \frac{1}{\sqrt{1-q}}\right)\right)$.

1) The operator $T_{\phi}$ is bounded and $\left\|T_{\phi}\right\| \leq\|\phi\|_{\infty}$.

2) By derivation under the integral sign and using (2), we have $T_{\bar{z}}=D_{q}$.

Theorem 2. If $\phi \in L^{\infty}\left(D\left(o, \frac{1}{\sqrt{1-q}}\right)\right)$ has compact support, then $T_{\phi}$ is a compact operator.

Proof. For $\phi \in L^{\infty}\left(D\left(o, \frac{1}{\sqrt{1-q}}\right)\right)$, we have

$$
\begin{aligned}
& \left\langle T_{\phi} \xi_{n}, \xi_{k}\right\rangle_{L^{2}}\left(D\left(o, \frac{1}{\sqrt{1-q}}\right), m_{q}\right) \\
& =\int_{D\left(o, \frac{1}{\sqrt{1-q}}\right)} T_{\phi} \xi_{n}(w) \overline{\xi_{k}(w)} \mathrm{d} m_{q}(w) .
\end{aligned}
$$

Since

$$
\begin{aligned}
& T_{\phi} \xi_{n}(w) \\
& =\int_{D\left(o, \frac{1}{\sqrt{1-q}}\right)} \phi(z) \xi_{n}(z) K_{q}(\bar{w}, \bar{z}) \mathrm{d} m_{q}(z) .
\end{aligned}
$$

Applying Fubini's theorem and Theorem 1, we obtain

$$
\begin{aligned}
& \left\langle T_{\phi} \xi_{n}, \xi_{k}\right\rangle_{L^{2}}\left(D\left(o, \frac{1}{\sqrt{1-q}}\right), m_{q}\right) \\
& =\left\langle\phi \xi_{n}, \xi_{k}\right\rangle_{L^{2}}\left(D\left(o, \frac{1}{\sqrt{1-q}}\right), m_{q}\right) .
\end{aligned}
$$

Thus,

$$
\begin{aligned}
& \sum_{n, k=0}^{\infty}\left|\left\langle T_{\phi} \xi_{n}, \xi_{k}\right\rangle_{L^{2}}\left(D\left(o, \frac{1}{\sqrt{1-q}}\right), m_{q}\right)\right|^{2} \\
& =\sum_{n, k=0}^{\infty}\left|\left\langle\phi \xi_{n}, \xi_{k}\right\rangle_{L^{2}}\left(D\left(o, \frac{1}{\sqrt{1-q}}\right), m_{q}\right)\right|^{2} .
\end{aligned}
$$

Since $\phi \in L^{\infty}\left(D\left(o, \frac{1}{\sqrt{1-q}}\right)\right)$ with compact support, there are positive constants $a$ and $K$ so that $|\phi(z)| \leq K$, a.e. and $\phi(z)=0$, for all $|z|>a$. Then for $k, n \in \mathbb{N}$, we get

$$
\begin{aligned}
& \left\langle\phi \xi_{n}, \xi_{k}\right\rangle_{L^{2}}\left(D\left(o, \frac{1}{\sqrt{1-q}}\right), m_{q}\right) \\
& =\frac{1}{\sqrt{[n]_{q} ![k]_{q} !}} \int_{|z| \leq a} \phi(z) z^{n} z^{-k} \mathrm{~d} m_{q}(z) .
\end{aligned}
$$

Thus, 


$$
\begin{aligned}
& \left|\left\langle\phi \xi_{n}, \xi_{k}\right\rangle_{L^{2}}\left(D\left(o, \frac{1}{\sqrt{1-q}}\right), m_{q}\right)\right| \\
& \leq \frac{K}{\sqrt{[n]_{q} ![k]_{q} !}} \int_{|z| \leq a}|z|^{n+k} \mathrm{~d} m_{q}(z) \\
& \leq \frac{K}{\sqrt{[n]_{q} ![k]_{q} !}} \int_{0}^{a^{2}} r^{(n+k) / 2} E_{q}(-q r) \mathrm{d}_{q} r \\
& \leq \frac{K a^{n+k}}{\sqrt{[n]_{q} ![k]_{q} !}} \int_{0}^{\frac{1}{1-q}} E_{q}(-q r) \mathrm{d}_{q} r .
\end{aligned}
$$

But from (1), we have

$$
\int_{0}^{\frac{1}{1-q}} E_{q}(-q r) \mathrm{d}_{q} r=\Gamma_{q}(1)=1 .
$$

Hence

$$
\left|\left\langle\phi \xi_{n}, \xi_{k}\right\rangle_{L^{2}}\left(D\left(o, \frac{1}{\sqrt{1-q}}\right), m_{q}\right)\right| \leq \frac{K}{\sqrt{[n]_{q} ![k]_{q} !}} a^{n+k} .
$$

Thus, we obtain

$$
\sum_{n, k=0}^{\infty}\left|\left\langle T_{\phi} \xi_{n}, \xi_{k}\right\rangle_{L^{2}}\left(D\left(o, \frac{1}{\sqrt{1-q}}\right), m_{q}\right)\right|^{2} \leq K^{2}\left[e_{q}\left(a^{2}\right)\right]^{2}<\infty .
$$

Then, $T_{\phi}$ is an Hilbert-Schmidt operator [7], and consequently it is compact.

\section{The Multiplication and Translation Operators on $\mathcal{F}_{q}$}

\subsection{The Derivative and Multiplication Operators on $\mathcal{F}_{q}$}

On $\mathcal{F}_{q}$, we consider the multiplication operator $Q$ given by

$$
Q f(z):=z f(z) .
$$

By straightforward calculation we obtain.

Lemma 2. $\left[D_{q}, Q\right]=D_{q} Q-Q D_{q}=\Lambda_{q}$, where $\Lambda_{q}$ is the q-shift operator given by

$$
\Lambda_{q} f(z):=f(q z) .
$$

This lemma is the $q$-analogous commutation rule of [1]. When $q \rightarrow 1^{-}$, then $\left[D_{q}, Q\right]$ tends to the identity operator $I$.

We now study the continuous property of the operators $\Lambda_{q}, D_{q}$ and $Q$ on $\mathcal{F}_{q}$.

Theorem 3. If $f \in \mathcal{F}_{q}$ then $\Lambda_{q} f, D_{q} f$ and $Q f$ belong to $\mathcal{F}_{q}$, and we have

1) $\left\|\Lambda_{q} f\right\|_{\mathcal{F}_{q}} \leq\|f\|_{\mathcal{F}_{q}}$,
2) $\left\|D_{q} f\right\|_{\mathcal{F}_{q}} \leq \frac{1}{\sqrt{1-q}}\|f\|_{\mathcal{F}_{q}}$,

3) $\|Q f\|_{\mathcal{F}_{q}} \leq \frac{1}{\sqrt{1-q}}\|f\|_{\mathcal{F}_{q}}$.

Proof. Let $f(z)=\sum_{n=0}^{\infty} a_{n} z^{n} \in \mathcal{F}_{q}$.

1) We have

$$
\Lambda_{q} f(z)=f(q z)=\sum_{n=0}^{\infty} a_{n} q^{n} z^{n},
$$

and from (3), we obtain

$$
\left\|\Lambda_{q} f\right\|_{\mathcal{F}_{q}}^{2}=\sum_{n=0}^{\infty}\left|a_{n}\right|^{2} q^{2 n}[n]_{q} ! \leq \sum_{n=0}^{\infty}\left|a_{n}\right|^{2}[n]_{q} !=\|f\|_{\mathcal{F}_{q}}^{2} .
$$

2) We have

$$
D_{q} f(z)=\sum_{n=1}^{\infty} a_{n}[n]_{q} z^{n-1}=\sum_{n=0}^{\infty} a_{n+1}[n+1]_{q} z^{n} .
$$

Then from (9), we get

$$
\left\|D_{q} f\right\|_{\mathcal{F}_{q}}^{2}=\sum_{n=0}^{\infty}\left|a_{n+1}\right|^{2}\left([n+1]_{q}\right)^{2}[n]_{q} !
$$

Since

$$
[n+1]_{q} !=[n+1]_{q}[n]_{q} !
$$

we obtain

$$
\left\|D_{q} f\right\|_{\mathcal{F}_{q}}^{2}=\sum_{n=0}^{\infty}\left|a_{n+1}\right|^{2}[n+1]_{q}[n+1]_{q} !
$$

and consequently,

$$
\left\|D_{q} f\right\|_{\mathcal{F}_{q}}^{2}=\sum_{n=0}^{\infty}\left|a_{n}\right|^{2}[n]_{q}[n]_{q} !
$$

Using the fact that $[n]_{q} \leq \frac{1}{1-q}$, we obtain

$$
\left\|D_{q} f\right\|_{\mathcal{F}_{q}} \leq \frac{1}{\sqrt{1-q}}\left[\sum_{n=0}^{\infty}\left|a_{n}\right|^{2}[n]_{q} !\right]^{1 / 2}=\frac{1}{\sqrt{1-q}}\|f\|_{\mathcal{F}_{q}} .
$$

3) On the other hand, since

$$
\text { Qf }(z)=\sum_{n=1}^{\infty} a_{n-1} z^{n},
$$

then

$$
\|Q f\|_{\mathcal{F}_{q}}^{2}=\sum_{n=1}^{\infty}\left|a_{n-1}\right|^{2}[n]_{q} !=\sum_{n=0}^{\infty}\left|a_{n}\right|^{2}[n+1]_{q} !
$$

By (10), we deduce

$$
\|Q f\|_{\mathcal{F}_{q}}^{2}=\sum_{n=0}^{\infty}\left|a_{n}\right|^{2}[n+1]_{q}[n]_{q} !
$$

Using the fact that $[n+1]_{q} \leq \frac{1}{1-q}$, we obtain 


$$
\|Q f\|_{\mathcal{F}_{q}} \leq \frac{1}{\sqrt{1-q}}\|f\|_{\mathcal{F}_{q}}
$$

We deduce also the following norm equality.

Theorem 4. 1) If $f \in \mathcal{F}_{q}$ then

$$
\|Q f\|_{\mathcal{F}_{q}}^{2}=\left\|D_{q} f\right\|_{\mathcal{F}_{q}}^{2}+\left\|\Lambda_{\sqrt{q}} f\right\|_{\mathcal{F}_{q}}^{2} .
$$

2) The operator $Q: \mathcal{F}_{q} \rightarrow \mathcal{F}_{q}$ is injective on $\mathcal{F}_{q}$.

Proof. Let $f(z)=\sum_{n=0}^{\infty} a_{n} z^{n} \in \mathcal{F}_{q}$.

1) By (13) and using the fact that $[n+1]_{q}=[n]_{q}+q^{n}$, we obtain

$$
\|Q f\|_{\mathcal{F}_{q}}^{2}=\sum_{n=0}^{\infty}\left|a_{n}\right|^{2}\left[[n]_{q}+q^{n}\right][n]_{q} !=\left\|D_{q} f\right\|_{\mathcal{F}_{q}}^{2}+\left\|\Lambda_{\sqrt{q}} f\right\|_{\mathcal{F}_{q}}^{2} .
$$

2) From (1), we have

$$
\|Q f\|_{\mathcal{F}_{q}}^{2} \geq\left\|\Lambda_{\sqrt{q}} f\right\|_{\mathcal{F}_{q}}^{2}
$$

Therefore $Q f=0$ implies that $f=0$. Then $Q: \mathcal{F}_{q} \rightarrow \mathcal{F}_{q}$ is injective continuous operator on $\mathcal{F}_{q}$.

Proposition 3. The operators $Q$ and $D_{q}$ are adjoint-operators on $\mathcal{F}_{q}$; and for all $f, g \in \mathcal{F}_{q}$, we have

$$
\langle Q f, g\rangle_{\mathcal{F}_{q}}=\left\langle f, D_{q} g\right\rangle_{\mathcal{F}_{q}} .
$$

Proof. Consider $f(z)=\sum_{n=0}^{\infty} a_{n} z^{n}$ and $g(z)=\sum_{n=0}^{\infty} b_{n} z^{n}$ in $\mathcal{F}_{q}$. From (9) and (12),

$$
\text { Qf }(z)=\sum_{n=1}^{\infty} a_{n-1} z^{n}, \quad D_{q} g(z)=\sum_{n=0}^{\infty} b_{n+1}[n+1]_{q} z^{n} .
$$

Thus from (4), we get

$$
\begin{aligned}
& \langle Q f, g\rangle_{\mathcal{F}_{q}}=\sum_{n=1}^{\infty} a_{n-1} \overline{b_{n}}[n]_{q} ! \\
& =\sum_{n=0}^{\infty} a_{n} \overline{b_{n+1}}[n+1]_{q} !=\left\langle f, D_{q} g\right\rangle_{\mathcal{F}_{q}},
\end{aligned}
$$

which gives the result.

\subsection{The Translation Operators on $\mathcal{F}_{q}$}

In this section we study a generalized translation operators on $\mathcal{F}_{q}$. We begin by the following definition.

Definition 3. For $f \in \mathcal{F}_{q}$ and $w, z \in D\left(o, \frac{1}{\sqrt{1-q}}\right)$, we define the $q$-translation operators on $\mathcal{F}_{q}$, by

$$
\tau_{z} f(w):=e_{q}\left(z D_{q}\right) f(w)=\sum_{n=0}^{\infty} D_{q}^{n} f(w) \frac{z^{n}}{[n]_{q} !} .
$$

For $w, z \in D\left(o, \frac{1}{\sqrt{1-q}}\right)$, the function $e_{q}$ satisfies the following product formula:

$$
\tau_{z} e_{q}(w)=e_{q}(z) e_{q}(w) .
$$

Proposition 4. Let $f(z)=\sum_{n=0}^{\infty} a_{n} z^{n} \in \mathcal{F}_{q}$ and $z, w \in D\left(o, \frac{1}{\sqrt{1-q}}\right)$. Then

$$
\tau_{z} f(w)=\sum_{n=0}^{\infty} a_{n} \sum_{k=0}^{n}\left(\begin{array}{l}
n \\
k
\end{array}\right)_{q} w^{n-k} z^{k} .
$$

Proof. Let $f(z)=\sum_{n=0}^{\infty} a_{n} z^{n} \in \mathcal{F}_{q}$. From (14), we have

$$
\tau_{z} f(w)=\sum_{n=0}^{\infty} \frac{D_{q}^{n} f(w)}{[n]_{q} !} z^{n} ; \quad w, z \in D\left(o, \frac{1}{\sqrt{1-q}}\right) .
$$

But from (5), we have

$$
D_{q}^{n} f(w)=\sum_{k=n}^{\infty} a_{k} \frac{[k]_{q} !}{[k-n]_{q} !} w^{k-n} .
$$

Thus we obtain

$$
\begin{aligned}
\tau_{z} f(w) & =\sum_{n=0}^{\infty} a_{n} \sum_{k=0}^{n} \frac{[n]_{q} !}{[k]_{q} ![n-k]_{q} !} w^{n-k} z^{k} \\
& =\sum_{n=0}^{\infty} a_{n} \sum_{k=0}^{n}\left(\begin{array}{l}
n \\
k
\end{array}\right)_{q} w^{n-k} z^{k} .
\end{aligned}
$$

Definition 4. For $f \in \mathcal{F}_{q}$ and $w, z \in D\left(o, \frac{1}{\sqrt{1-q}}\right)$, we define:

- The generalized multiplication operators on $\mathcal{F}_{q}$, by

$$
M_{z} f(w):=e_{q}(z Q) f(w)=\sum_{n=0}^{\infty} Q^{n} f(w) \frac{z^{n}}{[n]_{q} !} .
$$

- The generalized shift operators on $\mathcal{F}_{q}$, by

$$
S_{z} f(w):=e_{q}\left(z \Lambda_{q}\right) f(w)=\sum_{n=0}^{\infty} \Lambda_{q}^{n} f(w) \frac{z^{n}}{[n]_{q} !} .
$$

According to Theorem 3 we study the continuous property of the operators $\tau_{z}, M_{z}$ and $S_{z}$ on $\mathcal{F}_{q}$.

Theorem 5. If $f \in \mathcal{F}_{q}$ and $z \in D\left(o, \frac{1}{\sqrt{1-q}}\right)$, then $\tau_{z} f, M_{z} f$ and $S_{z} f$ belong to $\mathcal{F}_{q}$, and we have

$$
\text { 1) }\left\|\tau_{z} f\right\|_{\mathcal{F}_{q}} \leq e_{q}\left(\frac{|z|}{\sqrt{1-q}}\right)\|f\|_{\mathcal{F}_{q}} \text {, }
$$


2) $\left\|M_{z} f\right\|_{\mathcal{F}_{q}} \leq e_{q}\left(\frac{|z|}{\sqrt{1-q}}\right)\|f\|_{\mathcal{F}_{q}}$,

3) $\left\|S_{z} f\right\|_{\mathcal{F}_{q}} \leq e_{q}(|z|)\|f\|_{\mathcal{F}_{q}}$.

Proof. From (14) and Theorem 3 (2), we deduce

$\left\|\tau_{z} f\right\|_{\mathcal{F}_{q}} \leq \sum_{n=0}^{\infty}\left\|D_{q}^{n} f\right\|_{\mathcal{F}_{q}} \frac{|z|^{n}}{[n]_{q} !} \leq \sum_{n=0}^{\infty} \frac{|z|^{n}}{(1-q)^{n / 2}[n]_{q} !}\|f\|_{\mathcal{F}_{q}}$.

Therefore,

$$
\left\|\tau_{z} f\right\|_{\mathcal{F}_{q}} \leq e_{q}\left(\frac{|z|}{\sqrt{1-q}}\right)\|f\|_{\mathcal{F}_{q}},
$$

which gives the first inequality, and as in the same way we prove the second and the third inequalities of this theorem.

From Proposition 3 we deduce the following results.

Proposition 5. For all $f, g \in \mathcal{F}_{q}$, we have

$$
\begin{aligned}
& \left\langle M_{z} f, g\right\rangle_{\mathcal{F}_{q}}=\left\langle f, \tau_{\bar{z}} g\right\rangle_{\mathcal{F}_{q}}, \\
& \left\langle\tau_{z} f, g\right\rangle_{\mathcal{F}_{q}}=\left\langle f, M_{\bar{z}} g\right\rangle_{\mathcal{F}_{q}} .
\end{aligned}
$$

We denote by $R_{z}$ the following operator defined on $\mathcal{F}_{q}$ by

$$
\begin{aligned}
R_{z} & :=\tau_{\bar{z}} M_{z}-M_{\bar{z}} \tau_{\bar{z}} \\
& =e_{q}\left(\bar{z} D_{q}\right) e_{q}(z Q)-e_{q}(\bar{z} Q) e_{q}\left(z D_{q}\right) .
\end{aligned}
$$

Then, we prove the following theorem.

Theorem 6. For all $f \in \mathcal{F}_{q}$, we have

$$
\left\|M_{z} f\right\|_{\mathcal{F}_{q}}^{2}=\left\|\tau_{z} f\right\|_{\mathcal{F}_{q}}^{2}+\left\langle f, R_{z} f\right\rangle_{\mathcal{F}_{q}} .
$$

Proof. From Proposition 5, we get

$$
\begin{aligned}
\left\|M_{z} f\right\|_{\mathcal{F}_{q}}^{2} & =\left\langle f, \tau_{\bar{z}} M_{z} f\right\rangle_{\mathcal{F}_{q}}=\left\langle f,\left(M_{\bar{z}} \tau_{z}+R_{z}\right) f\right\rangle_{\mathcal{F}_{q}} \\
& =\left\|\tau_{z} f\right\|_{\mathcal{F}_{q}}^{2}+\left\langle f, R_{z} f\right\rangle_{\mathcal{F}_{q}} .
\end{aligned}
$$

\subsection{The Weyl Commutation Relations on $\mathcal{F}_{q}$}

Let $a, b \in D\left(o, \frac{1}{\sqrt{1-q}}\right)$. In this paragraph we establish

Weyl commutation relations between the translation operators $\tau_{a}$ and the multiplication operators $M_{b}$. These relations are realized on the Fock space $\mathcal{F}_{q}$.

Lemma 3. For $a, b \in D\left(o, \frac{1}{\sqrt{1-q}}\right)$, we have

1) $\left[D_{q}, Q^{n}\right]=[n]_{q} Q^{n-1} \Lambda_{q}, \quad n=1,2, \cdots$.
2) $\left[D_{q}, M_{b}\right]=b M_{b} \Lambda_{q}$.

Proof. 1) From Lemma 2, for $n=1,2, \cdots$, we deduce that

$$
\left[D_{q}, Q^{n}\right]=\sum_{k=0}^{n-1} Q^{k}\left[D_{q}, Q\right] Q^{n-k-1}=\sum_{k=0}^{n-1} Q^{k} \Lambda_{q} Q^{n-k-1} .
$$

Since

$$
\Lambda_{q} Q=q Q \Lambda_{q}
$$

we get

$$
\left[D_{q}, Q^{n}\right]=[n]_{q} Q^{n-1} \Lambda_{q} .
$$

Which proves the first equality.

2) We have

$$
\left[D_{q}, M_{b}\right]=\sum_{n=1}^{\infty} \frac{b^{n}}{[n]_{q} !}\left[D_{q}, Q^{n}\right] .
$$

Using (1), we obtain

$$
\begin{aligned}
{\left[D_{q}, M_{b}\right] } & =\sum_{n=1}^{\infty} Q^{n-1} \Lambda_{q} \frac{b^{n}}{[n-1]_{q} !} \\
& =b \sum_{n=0}^{\infty} Q^{n} \Lambda_{q} \frac{b^{n}}{[n]_{q} !}=b M_{b} \Lambda_{q} .
\end{aligned}
$$

Theorem 7. For $a, b \in D\left(o, \frac{1}{\sqrt{1-q}}\right)$, we have $\tau_{a} M_{b}=M_{b} \tau_{a} S_{a b}$.

Proof. From Lemma 3 (2), we have

$$
D_{q} M_{b}=M_{b}\left(D_{q}+b \Lambda_{q}\right) .
$$

Then, for $n=0,1,2, \cdots$, we deduce

$$
D_{q}^{n} M_{b}=M_{b}\left(D_{q}+b \Lambda_{q}\right)^{n} .
$$

Multiplying by $\frac{a^{n}}{[n]_{q} !}$ and summing, we get

$$
\tau_{a} M_{b}=M_{b} e_{q}\left(a D_{q}+a b \Lambda_{q}\right) .
$$

Since $D_{q} \Lambda_{q}=q \Lambda_{q} D_{q}$, from [5] we get

$$
e_{q}\left(a D_{q}+a b \Lambda_{q}\right)=e_{q}\left(a D_{q}\right) e_{q}\left(a b \Lambda_{q}\right)=\tau_{a} S_{a b},
$$

which completes the proof of the theorem.

Remark 4. If $q \rightarrow 1^{-}$, we obtain the classical commutation relations [8]:

$$
[D, Q]=I, \quad e^{a D} e^{b Q}=e^{a b} e^{b Q} e^{a D} ; \quad a, b \in \mathbb{C} .
$$

\section{References}

[1] V. Bargmann, "On a Hilbert Space of Analytic Functions 
and an Associated Integral Transform, Part I," Communications on Pure and Applied Mathematics, Vol. 14, No. 3, 1961, pp. 187-214. doi:10.1002/cpa.3160140303

[2] C. A. Berger and L. A. Coburn, "Toeplitz Operators on the Segal-Bargmann Space," Transactions of the American Mathematical Society, Vol. 301, 1987, pp. 813-829. doi:10.1090/S0002-9947-1987-0882716-4

[3] F. M. Cholewinski, "Generalized Fock Spaces and Associated Operators," Society for Industrial and Applied Mathematics, Journal on Mathematical Analysis, Vol. 15, No. 1, 1984, pp. 177-202. doi:10.1137/0515015

[4] G. H. Jackson, "On a $q$-Definite Integrals," Quarterly Journal of Pure and Applied Mathematics, Vol. 41, 1910, pp. 193-203.

[5] T. H. Koornwinder, "Special Functions and $q$-Commuting Variables," Fields Institute Communications, Vol. 14, 1997, pp. 131-166.

[6] G. Andrews, R. Askey and R. Roy, "Special Functions," Cambridge University Press, Cambridge, 1999.

[7] M. Naimark, "Normed Rings," Noordhoff, Groningen, 1959.

[8] T. Hida, "Brownian Motion," Springer-Verlag, Berlin, 1980 . 